\title{
Hot Stellar Populations in the M31 Bulge
}

\author{
HENRY C. FERGUSON \\ University of Cambridge, Institute of Astronomy \\ and \\ ARTHUR F. DAVIDSEN \\ Center for Astrophysical Sciences, The Johns Hopkins University
}

\begin{abstract}
We investigate the possibility that the Far-UV spectral energy distributions (SED's) of M31 and NGC 1399 differ only in the relative contribution from classical PAGB stars and in the amount of extinction. A good fit to the M31 spectrum is obtained for $E(B-V)=0.11$ if about $65 \%$ of the flux comes from PAGB stars and the rest from stars of the type producing the farUV emission in NGC1399. We speculate that the UV continuum in both galaxies is dominated by stars in post-horizontal branch phases of evolution, with a distribution of post-HB masses governed primarily by the metallicity and metallicity spread of the population. This hypothesis can qualitatively explain both the relative fluxes of UV rising branches in NGC1399 and M31 and the shapes of their SED's.
\end{abstract}

While post-asymptotic-giant-branch (PAGB) stars now seem unlikely to be the dominant contributor to the UV flux from the most metal-rich galaxies (Greggio \& Renzini 1990; Castellani \& Tornambè 1991; Ferguson et al. 1991), PAGB stars are known to be present in old metal-rich populations (as evidenced by the existence of planetary nebulae), and it is possible that they contribute significantly to the UV flux in galaxies with weaker upturns. To investigate this possibility, we have considered a simple model for the Hopkins Ultraviolet Telescope spectrum of M31 (Ferguson \& Davidsen 1992), in which a portion of the far-UV light comes from a single-mass population of PAGB stars, and a portion from the population (whatever it is) that is producing the far-UV emission in NGC 1399. We then adjust the relative proportions of the two populations, the PAGB mass, and the extinction to see whether we can achieve a good fit. This is by no means a unique or even realistic model, however it could indicate whether the concept of a composite population, with differing mixes of PAGB, PEAGB, EHB, and AGB Manqué stars (Greggio \& Renzini 1990), is at least viable.

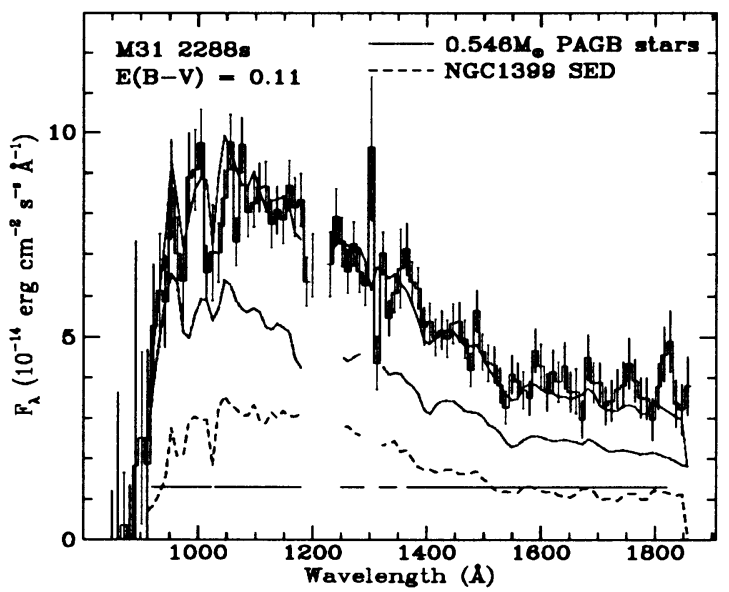

415
FIG. 1 - Best fit. PAGB + NGC 1399 model for the M31 spectrum. The M31 spectrum is shown, corrected for extinction assuming $E(B-V)=0.11$. The solid curve shows the best fit model for this extinction. The dashed curve shows the contribution from a NGC 1399-like stellar population, and the dotted curve shows the contribution from $0.546 M_{\odot}$ PAGB stars. 


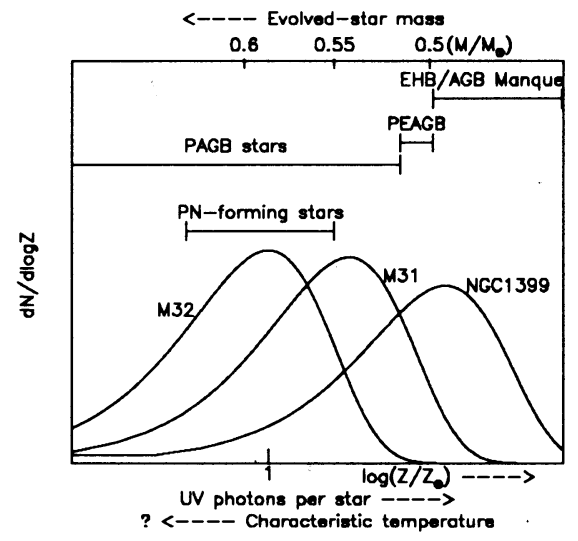

FIG.2 - Shows schematically the distribution of metallicities in M32, M31, and NGC 1399, galaxies with weak, moderate, and strong UV upturns, respectively. As metallicity increases, the core mass of each evolved star tends to decrease (Greggio \& Renzini 1990), the number of UV photons emitted over the lifetime of each star tends to increase, and the temperature at which most of the photons are emitted tends to decrease. Because of the rapid variation of the rate of evolution as a function of mass, PN sample only a narrow range of PAGB-star mass.

The best fit is shown in Fig. 1. Details of the fitting are discussed in our paper (Ferguson \& Davidsen 1992). The M31 SED can be fit with our simple model. If we hold $E(B-V)$ fixed, low-mass PAGB models are preferred, and contribute $\sim 65 \%$ of the flux at $1425 \AA$. Models with just an NGC 1399-like component are excluded with a high degree of confidence for any extinction. Our best estimate (Ferguson \& Davidsen 1992) is that central stars of planetary nebulae could account for no more than $1 \%$ of the flux, while the point sources detected by the HST FOC (King et al. 1992; Bertola 1992) could account for about $15 \%$. If the FOC calibration is correct, then the PAGB component in the M31 spectrum must come from very low mass $\left(<0.55 M_{\odot}\right)$ stars.

The result that M31 has a larger PAGB component than NGC 1399 is consistent with the hypothesis that the UV continuum in both galaxies is dominated by stars in $\mathrm{HB}$ and post-HB phases of evolution, with a distribution of masses governed by the metallicity and metallicity spread of the population. This is illustrated schematically in Fig. 2. The number of UV photons produced per star increases toward lower mass, while the characteristic temperature of the UV emitting population decreases, qualitatively explaining both the relative UV fluxes of NGC 1399 and M31 and the shapes of their SED's. Because planetary nebulae sample the high-mass end of the PAGB mass spectrum, we predict an anticorrelation in the number of PN per unit luminosity with $1550-V$ color, which we confirm with existing samples (Ferguson \& Davidsen 1992).

The HUT project is supported by NASA contract NAS5-27000 to the Johns Hopkins University. HCF is supported by SERC.

\section{References}

Bertola, F. 1992, preprint.

Castellani, M. \& Tornambè, A. 1991, ApJ, 381, 393

Ferguson, H. C. \& Davidsen, A. F. 1992, ApJ, submitted.

Ferguson, H. C., et al. , 1991, ApJ, 382, L69.

Greggio, L. \& Renzini, A. 1990, ApJ, 364, 35.

King, I., et al. , 1992, ApJ, 397, L35. 